\title{
Retrograde intubation: An alternative way for the management of difficult airway
}

\author{
Lama $\mathbf{P}^{1}$, Shrestha $\mathbf{B R}^{2}$ \\ ${ }^{1}$ Medical Officer, ${ }^{2}$ Assistant Professor, Department of Anesthesia and Intensive care, Kathmandu Medical College
}

\begin{abstract}
Inserting a retrograde wire into the pharynx through a cricothyroid puncture can facilitate tracheal intubation in difficult situations where either a flexible fiber-optic bronchoscope or an expert user of such a device is not available.Even in cases when fibropric can not be negotiated for the purpose, this method has been claimed to be useful to manage the airway. Some mouth opening is essential for the oral or nasal retrieval of the wire from the pharynx. Here, a case of post mandibular reconstructed wound infection required surgical debridement and plate removal from reconstructed lower mandible under general anesthesia. We retrieved the guide wire passed through a cricothyroid puncture and subsequently accomplished wire-guided oro-tracheal intubation. In the absence of a flexible fiber-optic bronchoscope, this technique is a very useful aid to intubate patients with limited mouth opening.
\end{abstract}

Key words: cricothyrotomy, guide wire, retrograde endotracheal intubation (REI), surgery.

$\mathrm{P}$ assing a retrograde catheter/wire through a cricothyroid puncture into the pharynx can facilitate tracheal intubation in difficult cases ${ }^{1,2}$. This technique of intubation has been applied successfully to secure difficult airways in elective and emergency situations $\mathrm{s}^{3,4}$. However, some degree of mouth opening is essential to bring out the catheter/wire from the oropharynx. We performed oro-tracheal intubation in patient with postmandibular reconstructed wound infection with extremely limited mouth opening. The following case report describes the technique in detail.

\section{Case report}

A 20 year man, ASA I with a history of electric burn injury in lower mandibular area presented with wound infection and plate coming out from the reconstructed lower mandibular area. Previous reconstruction of lower mandibular surgery was complicated by infection and sloughing of skin and subcutaneous tissue of lower half of face and the anterior neck was grossly scarred. He was diagnosed as post reconstructed mandibular wound infection and was planned for debridement and removal of plate under general anaesthesia.

Mallampati orophayngeal view was difficult to assess due to scarring and limited mouth opening. Thyromental distance and sternomental distance could not be measured accurately due to the swelling, laceration and scarring of reconstructed lower mandibular area.
The patient had been undergoing stepwise reconstructive surgery of lower mandibular area in other centers for past four years. Multiple tracheostomies were performed during the surgery for airway management. The patient had undergone scheduled reconstructive surgery. The patient had undergone retrograde endotracheal intubation during last surgery due to failed blind nasal intubation and difficult nasotracheal intubation.

For this operation, it was again anticipated that the trachea would be impossible to intubate through oropharynx with the help of laryngoscope. In the absence of a flexible fiber-optic bronchoscope, we planned to place a guide wire through a cricothyroid puncture and bring it out from the hypopharynx and perform retrograde endotracheal intubation. In this difficult airway situation, we chose to perform the procedure in the conscious patient. The procedure was explained to the patient and his attendents. Possible failure of the technique requiring further interventions such as blind intubation attempts and tracheostomy were also explained and consent was taken for the procedure and tracheostomy. The night before surgery, the patient was premedicated with Alprazolam $0.25 \mathrm{mg}$, Ranitidine $150 \mathrm{mg}$ and Metoclopromide 10mg per oral.

Correspondence

Dr. Babu Raja Shrestha

Department of anaesthesia and Intensive care

Kathmandu Medical College Teaching Hospital, Sinamangal

E-mail: barashrestha@yahoo.co.in 
At operation theatre, difficult airway equipment was assembled and 20 gauge $(G)$ intravenous cannula was placed in the left arm of the patient and he was monitored. Lignocaine $60 \mathrm{mg}$ IV was given and Lignocaine 10\% spray was sprayed through the oropharynx. After infiltration of local anesthetic, $16 \mathrm{G}$ cannula was placed in trachea through cricothyroid membrane. Coughing on insertion, free aspiration of air through the syringe with normal saline and the presence of palpable flow of air through the cannula on exhalation confirmed the position.

After cricothyroid membrane was punctured, the guide wire (malleable end) was inserted through the cannula and was pushed upward and orally retrieved from pharynx without any difficulty. Angiography or urology

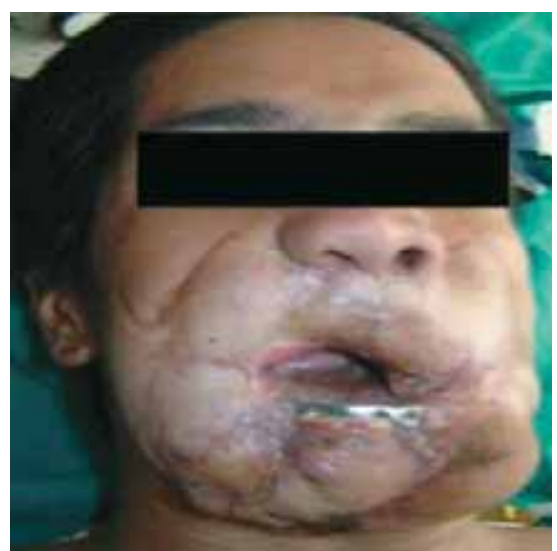

Fig 1: Limited (maximal) mouth opening and plate coming out of the lower mandibular area.

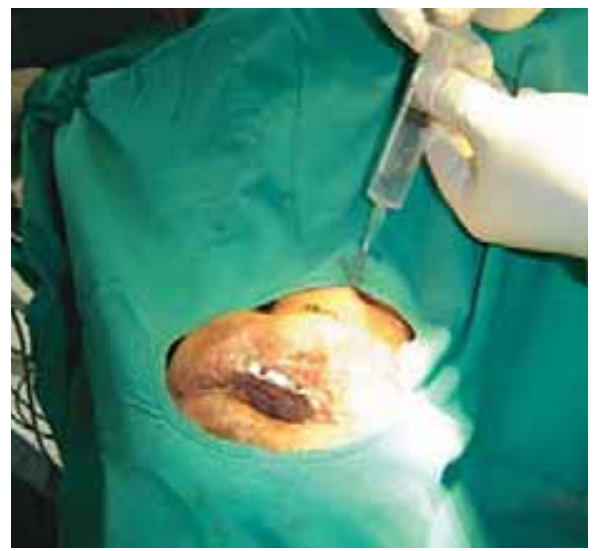

Fig 3: Air bubbles coming out in the syringe containing normal saline confirming the position of trachea. wire can be easily used for the purpose. The endotracheal tube was then inserted over the guide wire and pushed into the trachea through oropharynx fixing the distal end of the guide wire. The endotracheal positioning of the tube was confirmed after checking for bilateral air entry and confirmation by capnography. Then guide wire was removed from the oropharynx while $6.5 \mathrm{~mm}$ cuffed endotracheal tube was maintained in tracheal lumen. The intraoperative period was uneventful. After the completion of surgery the patient was shifted to Intensive Care Unit with the endotracheal tube in situ and for elective ventilation. The patient was extubated on the $2^{\text {nd }}$ postoperative day and shifted to the plastic and reconstruction ward and was discharged on the $5^{\text {th }}$ postoperative day.

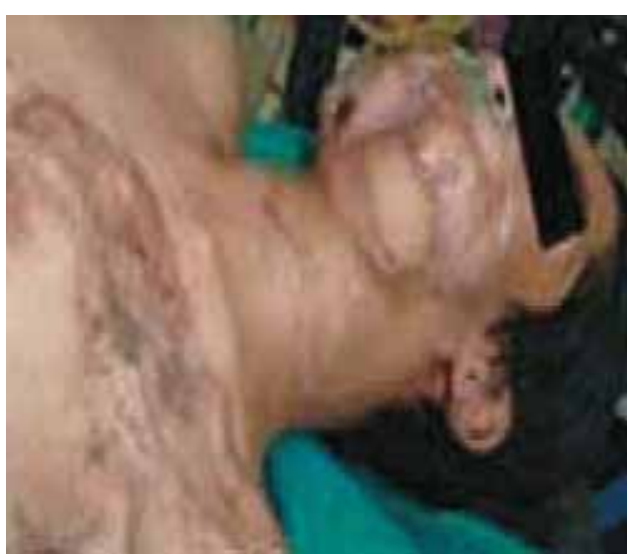

Fig 2: Multiple scarring of the anterior neck and deformed lower mandibular area with swelling and scarring.

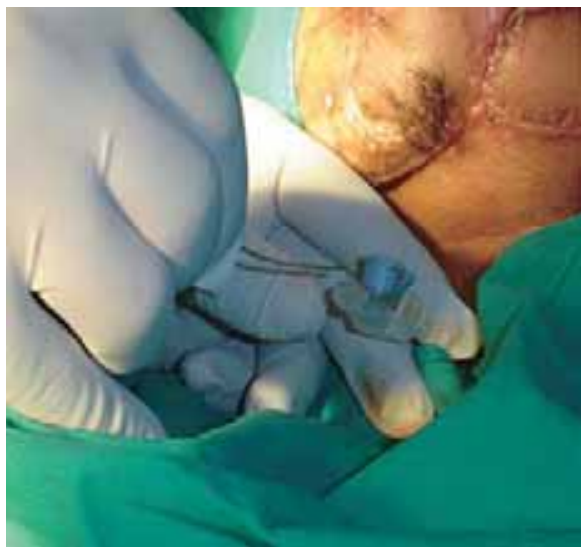

Fig 4: Passing guide wire into $16 \mathrm{G}$ cannula through the cricothyroid membrane. 


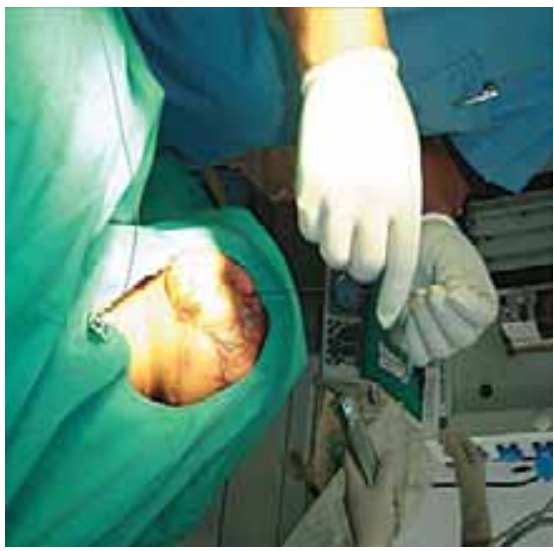

Fig 5: Retrieval of guide wire through the oropharynx.

\begin{abstract}
Discussion
The patient presented with multiple problems for airway management like difficult direct laryngoscopy (reduced mouth opening, edematous and scarred lower mandibular area), difficult laryngeal mask airway placement (limited mouth opening), history of failed blind nasal intubation, scarring of the anterior neck and possible tracheal stenosis due to repeated tracheostomy.
\end{abstract}

The ASA difficult airway algorithm suggests difficult cases should be assessed for ease of mask ventilation, ease of intubation and ease of direct tracheal asses. In this case all three routes were anticipated to be difficult.

Described in the early $1960^{3,4}$, retrograde endotracheal intubation is an alternative technique for difficult airway management ${ }^{5,6}$. However, retrograde endotracheal intubation is usually available ${ }^{7}$, it is not often used. The study conducted by the department of emergency medicine Loma Linda University of school of medicine included only eight cases of retrograde endotracheal intubation out of 1368 cases of intubation in over eight years. The indication were head and facial trauma, cervical spine trauma, upper airway blockade above the vocal cord and unable to visualize the vocal cord. The complications were inability to locate the cricothyroid membrane $(n=2)$, inability to pass the endotracheal tube through the vocal cord $(n=1)$, prolonged procedure time $(n=4)$ and success rate was four out of eight cases. Skill of the operator and difficult airway anatomy might influence the success rate of the REI ${ }^{8}$.

Another study conducted by Francois lenfet on the fresh human cadavers had the success rate of $70 \%{ }^{9}$.

Publications about the complications of REI are sparse. However, potential complications included hypoxemia,

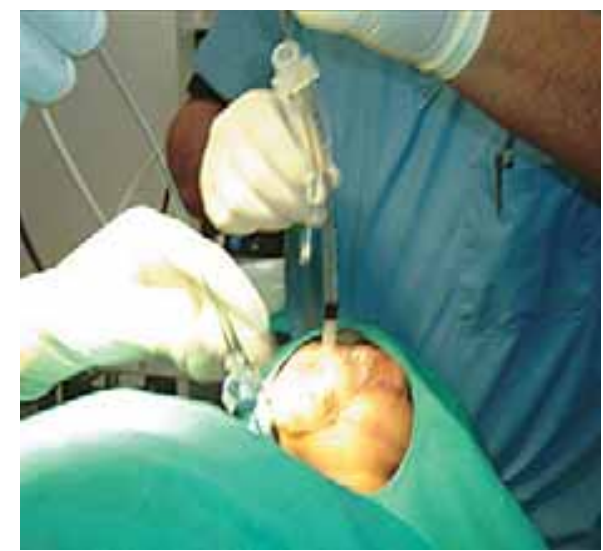

Fig 6: Endotracheal tube $6.5 \mathrm{~mm}$ was being inserted onto the guidewire and pushed into the tracheal lumen through the oropharynx.

sore throat, cough, laryngospasm/bronchospasm ${ }^{5}$, development of hematoma, incorrect site of tube placement, procedure time greater than or equal to three minutes, unsuccessful tube placement, laryngeal fracture with permanent dystonia, pneumomediastinum ${ }^{9}$, subcutaneous emphysema, infection and bleeding ${ }^{10-17}$. Retained wire ${ }^{18}$, nasal mucosal injuries as signified by the presence of epistaxis ${ }^{17,18}$, cord ulceration, recurrent superior laryngeal nerve injury, infection of the wound, and respiratory tract infection were also noted. Indeed, any bleeding disorders or coagulopathy should be investigated first to avoid the grave complication of bleeding and haematoma.

Ideally, a flexible fiber-optic bronchoscope is the alternative choice for tracheal intubation of such patients. A high purchase and maintenance cost of fiber-optic devices has been the cause of unavailability of fiber-optic bronchoscope in Operation theatre of resource poor centers.

As a retrograde guide wire one can use any simple atraumatic wires for this purpose like epidural catheter, angioplasty or urology guide wire.Multilumen catheter has also been described as an anterograde guide over the retrograde wire to firmly fix the endotracheal tube deep down into the trachea through the laryngeal inlet and the same to be used for the jet ventilation purpose ${ }^{19}$.

Tracheostomy would have been an appropriate option to secure the airway under these circumstances if multistage reconstructive surgery was planned, with challenges of a difficult airway each time and significant risk of prolonged postoperative airway compromise resulting from soft tissue edema. However tracheostomy was not performed due to the single staged surgery, patient's denial, possible fear of tracheal stenosis, scarring of the anterior neck of the patient. 


\section{Conclusion}

In the absence of a fiber-optic bronchoscope and relative contraindication of blind nasal intubation and tracheostomy, guide wire guided retrograde endotracheal intubation can be a suitable and safe alternative for airway management of difficult airway.

\section{References}

1. Cobley M, Vaughan RS. Recognition and management of difficult airway. Br J Anesthesia 1992;68:90-7.

2. Karkouti K, Rose DK, Wigglesworth D, Cohen MM. Predicting a difficult intubation: multivariable analysis. Can J Anesthesia 2000;47:730-9.

3. Butler FS, Cirillo AA. Retrograde endotracheal intubation. Anesth Analg 1960;39(4):333-8.

4. Waters DJ. Guided blind endotracheal intubation: for patients with deformities of the upper airway. Anesthesia 1963;18:158-62.

5. American Society of Anesthesiologists. Practice guidelines for management of the difficult airway: An updated Report by the American Society of Anesthesiologists Task Force on Management of the Difficult Airway. Anesthesiology 2003;98:1269-77.

6. SFAR: Intubation difficile, expertise collective. Ann Fr Anesth Reanim 1996;15:207-14.

7. Levitan RM, Kush S, Hollander JE. Devices for difficult airway management in academic emergency departments: results of a national Survey. Ann Emerg Med 1999;33(6):694-8.

8. Michelle G, Mathew JM, Steren MG. Retrograde endotracheal intubation - an investigation of indication, complication and patient outcomes. American journal of emergency medicine 2005;23:123-6.
9. Francois L, Mehdi B. Comparison of two techniquesfor retrograde endotrachealintubation in fresh human cadaver. Anesthesiology 2006 Jan;104(1):48-51.

10. McNamara RM. Retrograde intubation of the trachea. Ann Emerg Med 1987;16(6):680- 2.

11. McGill J, Clinton JE, Ruiz E. Cricothyrotomy in the emergency department. Ann Emerg Med 1982;11(7):361-4.

12. Wijesinghe $\mathrm{H}$, Gough J. Complications of a retrograde intubation in a trauma patient. Acad Emerg Med 2000;7(11):1267-71.

13. Bowes WA, Johnson JO. Pneumomediastinum after planned retrograde fiber optic intubation. Anesth Analg 1994;78:795-7.

14. Beebe DS, Tran P, Belani KG, Adams GL. Pretrachealabscess following retrograde tracheal intubation. Anesthesia 1995 May;50(5):470.

15. Parmet J, Metz S. Retrograde endotracheal intubation: an underutilized tool for management of the difficult airway. Contempt Surgery1996;49(5):300-6.

16. Sanchez TF. Retrograde intubation. Anesthesia Clin North Am 1995;13:439-76.

17. Akinyemi OO. Complications of guided blind endotracheal intubation. Anesthesia 1979;34:590-2.

18. Loh KS, Irish JC. Traumatic complications of intubation and other airway management procedures. Anesthesia Clin North Am 2002;20(4):953-69.

19. Dhara SS. Retrograde intubation - a facilitated approach. Br J Anaesth. 1992 Dec;69(6):631-3. 